\title{
PSMA-Ligand Uptake Can Serve as a Novel Biomarker in Primary Prostate Cancer to Predict Outcome After Radical Prostatectomy
}

Hui Wang ( $\sim$ huihui.wang@tum.de)

Klinikum rechts der Isar der Technischen Universitat Munchen Nuklearmedizinische Klinik https://orcid.org/0000-0003-1494-6668

Thomas Amiel

Klinikum rechts der Isar der Technischen Universitat Munchen, Urologie

Christoph Würnschimmel

Universitatsklinikum Hamburg-Eppendorf, Martini-Klinik

Thomas Langbein

Klinikum rechts der Isar der Technischen Universitat Munchen Nuklearmedizinische Klinik

\section{Katja Steiger}

Institut für Allgemeine Pathologie und Pathologische Anatomie der Technischen Universität München

Isabel Rauscher

Klinikum rechts der Isar der Technischen Universitat Munchen Nuklearmedizinische Klinik

Thomas Horn

Klinikum rechts der Isar der Technischen Universitat Munchen, Urologie

Tobias Maurer

Universitatsklinikum Hamburg-Eppendorf, Martini-Klinik

\section{Wolfgang Weber}

Klinikum rechts der Isar der Technischen Universitat Munchen Nuklearmedizinische Klinik Hans-Juergen Wester

Technische Universitat Munchen, Pharmazeutische Radiochemie

\section{Karina Knorr}

Klinikum rechts der Isar der Technischen Universitat Munchen Nuklearmedizinische Klinik

\section{Matthias Eiber}

Klinikum rechts der Isar der Technischen Universitat Munchen Nuklearmedizinische Klinik

\section{Research Article}

Keywords: Biochemical recurrence, 68Ga-PSMA-11 PET, miTNM classification, prostate cancer

Posted Date: June 22nd, 2021 
DOl: https://doi.org/10.21203/rs.3.rs-603830/v1

License: (c) (1) This work is licensed under a Creative Commons Attribution 4.0 International License. Read Full License

Version of Record: A version of this preprint was published at EJNMMI Research on August 21st, 2021. See the published version at https://doi.org/10.1186/s13550-021-00818-2. 


\section{Abstract}

\section{Background}

The prostate-specific membrane antigen (PSMA) is a relevant target in prostate cancer and immunohistochemistry studies showed associations with outcome.

PSMA-ligand positron emission tomography (PET) is increasingly used for primary prostate cancer staging and the molecular imaging TNM classification (miTNM) standardizes its reporting. We aimed to investigate the potential of PET-imaging to serve as a noninvasive imaging biomarker to predict disease outcome in primary prostate cancer after radical prostatectomy (RP).

Methods

In this retrospective analysis, 186 primary prostate cancer patients treated with RP who had undergone a ${ }^{68} \mathrm{Ga}$-PSMA-11 PET up to three months prior to the surgery were included. Maximum standardized uptake value $\left(S U V_{\text {max }}\right)$, SUV mean, tumor volume $(T V)$ and total lesion $(T L)$ were collected from PET-imaging. Moreover, clinicopathological information, including age, serum prostate-specific antigen (PSA) level, and pathological characteristics were assessed for disease outcome prediction. A stage group system for PET-imaging findings based on the miTNM framework was developed.

Results

At a median follow-up after RP of 38 months (interquartile range (IQR): 22-53), biochemical recurrence (BCR) was observed in 58 patients during the follow-up period. A significant association between a positive surgical margin and miN status (miN1 vs. miN0, odds ratio (OR): $5.428, p=0.004$ ) was detected. miT status (miT $\geq 3$ a vs. miT<3, OR: 2.696, $p=0.003$ ) was identified as an independent predictor for Gleason score $(G S) \geq 8$. Multivariate Cox regression analysis indicated that PSA level (hazard ratio (HR): 1.024, $p=0.014$ ), advanced $G S$ ( $G S \geq 8$ vs. $G S<8, H R: 3.253, p<0.001)$ and miT status (miT $\geq 3$ a vs. miT $<3$, HR: $1.941, p=0.035$ ) were independent predictors for BCR. For stage I disease as determined by PETimaging a shorter BCR-free survival was observed in the patients with higher SUV $\mathrm{max}_{\text {ax }}$ (IA vs. IB stage, logrank, $p=0.022$ ).

Conclusion

Preoperative miTNM classification from ${ }^{68} \mathrm{Ga}$-PSMA-11 PET correlates with postoperative GS, surgical margin status and time to BCR. The association between miTNM staging and outcome proposes ${ }^{68} \mathrm{Ga}-$ PSMA-11 PET as a novel non-invasive imaging biomarker and potentially serves for ancillary pretreatment stratification. Prospective studies with larger cohort are necessary to fully determine its use including primary prostate cancer patients with different treatments and risk categories and late-stage patients. 


\section{Background}

Approximately $30 \%$ to $40 \%$ of prostate cancer patients will fail primary treatment requiring further disease management $[1,2]$. Traditional risk factors, including preoperative serum prostate-specific antigen (PSA) level [3-6], pathological stage [7] and Gleason score (GS) [5-9] are widely used for biochemical recurrence (BCR) prediction. However, there is growing interest in identifying novel biomarkers to improve BCR prediction accuracy of prostate cancer patients after radical prostatectomy (RP).

In the last few years positron emission tomography (PET) probes targeting prostate-specific membrane antigen (PSMA) has significantly improved detection and localization of disease in primary and recurrent prostate cancer [1, 10-12]. PSMA is a type II integral membrane glycoprotein with folate hydrolase activity, internalization after activation and is encoded by the FOLH1 gene [13, 14]. PSMA expression increases progressively in higher-grade prostate tumor cells and metastatic lesions $[15,16]$.

Increased PSMA expression in immunohistochemistry was more often observed in pathological stage III or IV tumors $(51 \%)$ compared to stage I and II tumors $(32 \%, p=0.029)$ [17]. High level PSMA expression in immunohistochemistry was associated with a higher risk of BCR and overall survival in several studies $[16,17]$. Finally, expression of membranous PSMA is also associated with higher rates of defective deoxyribonucleic acid (DNA) damage repair gene [18].

In the last two decades, for different tumor entities results from imaging were introduced as non-invasive quantitative biomarkers. For fluorodeoxyglucose (FDG)-PET-imaging, Wieder et al. have demonstrated that mean standardized uptake value $\left(\mathrm{SUV}_{\text {mean }}\right)$ can be used to preoperatively predict histopathological response in esophageal squamous cell carcinoma (ESCC) patients. A decrease in $S_{\text {mean }}$ of $44 \% \pm 15 \%$ from responders and $21 \% \pm 14 \%$ from non-responders $(p=0.0055)$ was observed after radiochemotherapy [19]. For metastatic castration-resistant prostate cancer (mCRPC) the bone scan index (BSI) [20] and quantitative parameter from PET have been reported to serve as potential predictive biomarkers for bone tumor burden [21, 22].

To standardize reporting of PSMA-targeted PET-imaging a unified molecular-imaging TNM classification (miTNM, version 1.0) has been recently introduced [23]. It is envisioned that its system classifying tumor extent similar to the pathological TNM-system might serve as qualitative imaging biomarker potentially stratifying disease outcome.

The aim of our retrospective analysis was to investigate the potential of quantitative and qualitative parameters from ${ }^{68} \mathrm{Ga}$-PSMA-11 PET to serve as non-invasive imaging biomarkers to predict BCR in primary prostate cancer after RP allowing for ancillary preoperative risk stratification.

\section{Methods}

\section{Patient selection}


We screened the institutions' database for all patients who underwent ${ }^{68} \mathrm{Ga}$-PSMA-11 PET imaging maximum 3 months prior the RP between January 2013 and August 2017. All patients who received neoadjuvant therapy prior to RP, had PSA-persistence after RP or in whom follow-up data were missing were excluded. Finally, 186 patients with D'Amico intermediate- to high-risk primary prostate cancer were included in this retrospective study (Supplementary Fig. 1). Table 1 summarizes the clinical and histopathological characteristics. BCR was defined as a serum PSA level rising above $0.2 \mathrm{ng} / \mathrm{ml}$. The primary endpoint was time to BCR. The time to BCR was calculated from the date of surgery. The retrospective analysis has been approved by the Ethics Committee of the Technical University Munich (750/20 S-KH).

\section{Imaging protocol}

The synthesis of ${ }^{68} \mathrm{Ga}$-PSMA-11 [24] was described previously [25]. Patients were intravenously injected with a median of $139 \mathrm{MBq}$ of ${ }^{68} \mathrm{Ga}-\mathrm{PSMA}-11$ (interquartile range (IQR): 112-156). PET acquisition was started at a median of 54 min (IQR: 49-65) after the tracer injection. Nighty-three patients underwent ${ }^{68} \mathrm{Ga}$ PSMA-11 PET/computed tomography (CT) using a Biograph mCT flow scanner (Siemens Medical Solutions, Erlangen, Germany), and 93 patients underwent ${ }^{68} \mathrm{Ga}-\mathrm{PSMA}-11 \mathrm{PET} /$ magnetic resonance imaging (MRI) using an integrated whole-body PET/MRI system (Siemens Biograph mMR, Erlangen, Germany). Details on PET/CT and PET/MRI acquisition were described previously $[26,27]$.

\section{Imaging analysis}

${ }^{68} \mathrm{Ga}-\mathrm{PSMA}-11 \mathrm{PET} / \mathrm{CT}$ and ${ }^{68} \mathrm{Ga}$-PSMA-11 PET/MRI images were evaluated by one nuclear medicine physician blinded to the postoperative histopathological results. All lesions were reannotated by two experienced board-certified nuclear medicine physicians. Any focal or diffuse tracer uptake in the prostate or extra-prostatic lesions above the surrounding background and not associated with physiological uptake was considered suspicious for malignancy. One circular region in transaxial slices was drawn over the prostate and over every extra-prostatic lesion automatically adapted to a three-dimensional volume of interest (VOI) using Syngo.Via (Siemens Healthineers, Erlangen, Germany) at $40 \%$ isocontour. Typical pitfalls in PSMA-ligand PET including low to moderate PSMA uptake correlated with osteoblastic changes (i.e., fractures or degenerative changes), celiac, and ganglia were taken into consideration [28]. SUV $_{\text {max }}, S_{\text {SU }}$ mean, tumor volume (TV), and total lesion (TL) of every VOI were calculated. Prostatic and extra-prostatic lesions were classified according to miTNM classification [23].

Similar to the structure of the American Joint Committee on Cancer (AJCC) Prostate Cancer Prognostic Stage Groups [29], we established a stage group system using the different grades from the miTNM staging system (Table 2). To allow the discrimination into different risk groups based on the

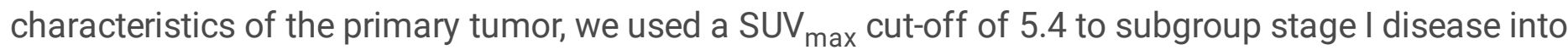
stage IA and IB. The cut-off was derived from a recent study proposing it the optimal cut-off to distinguish between $\mathrm{GS} \leq 7 \mathrm{a}$ and $\mathrm{GS} \geq 7 \mathrm{~b}[30]$. 
Table 2. Proposed miTNM stage groups for ${ }^{68} \mathrm{Ga}-\mathrm{PSMA}-11$ PET.

\begin{tabular}{lllll} 
Stage Group & miT & miN & miM & SUV $_{\max }$ \\
\hline IA & 2 & 0 & 0 & $<5.4$ \\
\hline IB & 2 & 0 & 0 & $\geq 5.4$ \\
\hline IIA & 3 & 0 & 0 & Any \\
\hline IIB & 4 & 0 & 0 & Any \\
\hline III & Any & 1 and 2 & 0 & Any \\
\hline IV & Any & Any & 1 & Any
\end{tabular}

PET = positron emission tomography; PSMA = prostate-specific membrane antigen; SUV = standardized uptake value.

\section{Statistical analysis}

Descriptive statistics were used to display continuous variables as the median and IQR with $25^{\text {th }}$ and $75^{\text {th }}$ percentiles (Q1-Q3), mean \pm standard deviation (SD), as well as percentages. The association between pathological results and ${ }^{68} \mathrm{Ga}-\mathrm{PSMA}-11$ PET findings was investigated with uni- and multivariate Logic regression analyses, and the corresponding odds ratios (OR) and 95\% confidence intervals (CI) were calculated. Postoperative BCR-free survival was estimated using the Kaplan-Meier method and compared between groups using the Log-rank test. Moreover, uni- and multivariable Cox regression analysis were performed to determine the ability of clinicopathological factors and ${ }^{68} \mathrm{Ga}-\mathrm{PSMA}-11$ PET findings to predict BRC after RP, and the corresponding hazard ratios (HR) and $95 \% \mathrm{Cl}$ were calculated. The multivariable model only included parameters with a significant association on univariable analysis. A $p$ value of 0.05 was used as the cut-off for statistical significance.

Given its low sample size $(n=7)$ the miM1 subgroup was excluded for univariable and multivariable analysis.

Statistical evaluation was performed with IBM SPSS Statistics Version 20 (Armonk, NY, USA), and the figures were generated using GraphPad Prism Version 8 (San Diego, California, USA).

\section{Results}

\section{Histopathological patient characteristics}

On post-operative histopathology, a total of $133(71.5 \%)$ patients had a $G S<8$, and $53(28.5 \%)$ of the patients had a GS 8 or 9 . Lymph node metastases were detected in $32(17.2 \%)$ patients. pT3a, pT3b and pT4 disease was present in 49 (26.3\%), 44 (23.7\%) and 1 (0.5\%) patient, respectively. Twenty-eight 
(15.6\%) patients had positive surgical margins (R1) (Table 1). At a median follow-up of 38 months (IQR: 22-53), BCR was observed in 58 (31.2\%) patients during the follow-up period.

Table 1. Patient characteristics.

\begin{tabular}{|c|c|}
\hline Characteristic & Patients \\
\hline Age (yr), median (IQR), $n=186$ & $68(61-72)$ \\
\hline iPSA (ng/ml), median (IQR), n=184a & $9.7(6.5-15.1)$ \\
\hline Administered ${ }^{68} \mathrm{Ga}-\mathrm{PSMA}-11$ activity (MBq), median (IQR), $\mathrm{n}=185^{\mathrm{b}}$ & $139(112-156)$ \\
\hline Time PET to RP (day), median (IQR), n=186 & $26(13-46)$ \\
\hline \multicolumn{2}{|l|}{ Gleason score in surgical specimen, no. (\%), n=186 } \\
\hline 6 & $11(5.9 \%)$ \\
\hline $7 a$ & $63(33.9 \%)$ \\
\hline $7 \mathrm{~b}$ & $59(31.7 \%)$ \\
\hline 8 & $28(15.1 \%)$ \\
\hline 9 & $25(13.4 \%)$ \\
\hline \multicolumn{2}{|l|}{ Pathological stage, no. (\%), n=186 } \\
\hline \multicolumn{2}{|l|}{ pT status } \\
\hline $2 a$ & $11(5.9 \%)$ \\
\hline $2 b$ & $10(5.4 \%)$ \\
\hline $2 c$ & $71(38.2 \%)$ \\
\hline $3 a$ & $49(26.3 \%)$ \\
\hline $3 b$ & $44(23.7 \%)$ \\
\hline 4 & $1(0.5 \%)$ \\
\hline \multicolumn{2}{|l|}{ pN status } \\
\hline 0 & $154(82.8 \%)$ \\
\hline 1 & $32(17.2 \%)$ \\
\hline
\end{tabular}

Surgical margin, no. (\%), $\mathrm{n}=180^{\mathrm{C}}$

Negative

$152(84.4 \%)$

Positive

$28(15.6 \%)$ 
a: iPSA of two patients were unavailable; ${ }^{\text {b: }}$ the injected dose of ${ }^{68} \mathrm{Ga}-\mathrm{PSMA}-11$ from one patient was unavailable; ${ }^{c}$ : the status of surgical margin from six patients were unavailable.

iPSA = initial PSA; IQR = interquartile range; $\mathrm{PET}=$ positron emission tomography; $\mathrm{PSA}=$ prostate-specific antigen; $\mathrm{RP}=$ radical prostatectomy.

\section{${ }^{68}$ Ga-PSMA-11 PET findings}

A. miTNM staging and miTNM stage groups

In $67.2 \%(n=125)$ of patients the primary tumor was classified as miT2, 90.3\% ( $n=168)$ were classified as miN0, 3.8\% $(n=7)$ were classified as miN1, 5.9\% $(n=11)$ were classified as miN2, and $96.2 \%(n=179)$ were classified as miM0. ${ }^{68} \mathrm{Ga}$-PSMA-11 PET findings (SUV max, SUV $\left._{\text {mean }}, \mathrm{TV}, \mathrm{TL}\right)$ of prostatic lesions were analyzed with 183 patients because three patients were reported negative PSMA prostate cancer. Table 3 lists information from ${ }^{68} \mathrm{Ga}-\mathrm{PSMA}-11 \mathrm{PET}$.

Table 3. ${ }^{68} \mathrm{Ga}-\mathrm{PSMA}-11$ PET findings. 
miTNM classification, no. (\%), $\mathrm{n}=186$

miT status

\begin{tabular}{cl}
\hline $2 \mathrm{u}$ & $73(39.2 \%)$ \\
\hline $2 \mathrm{~m}$ & $52(28 \%)$ \\
\hline $3 \mathrm{a}$ & $27(14.5 \%)$ \\
\hline $3 \mathrm{~b}$ & $24(12.9 \%)$ \\
\hline 4 & $10(5.4 \%)$ \\
\hline $\mathrm{miN}$ status & \\
\hline 0 & $168(90.3 \%)$ \\
\hline 1 & $7(3.8 \%)$ \\
\hline 2 & $11(5.9 \%)$ \\
\hline $\mathrm{miM}$ status & $179(96.2 \%)$ \\
\hline 0 & $3(1.6 \%)$ \\
\hline $1 \mathrm{a}$ & $4(2.2 \%)$ \\
\hline $1 \mathrm{~b}$ & \\
\hline
\end{tabular}

PSMA-PET findings of prostatic lesions, median (IQR), $n=183^{\mathrm{a}}$

\begin{tabular}{ll}
\hline SUV $_{\text {max }}$ & $10.6(6.4-18.9)$ \\
\hline SUV $_{\text {mean }}$ & $6.2(3.2-11.0)$ \\
\hline TV & $3.9(1.7-10.5)$ \\
\hline TL & $24.7(15.9-44.4)$
\end{tabular}

a: three patients had PSMA negative prostate cancer. miTNM of these patients were classified based on MRI images.

$\mathrm{IQR}=$ interquartile range; $\mathrm{PET}$ = positron emission tomography; PSMA = prostate-specific membrane antigen; SUV = standardized uptake value; $T L=$ total lesion; TV = tumor volume.

Based on the proposed stage group system combining the miTNM staging by and SUV max $20(10.8 \%)$, 96 (51.6\%), 40 (21.5\%), 7 (3.8\%), 16 (8.6\%) and 7 (3.8\%) into the stage groups IA, IB, IIA, IIB, III and IV, respectively. 
B. Correlation of $68 \mathrm{Ga}-\mathrm{PSMA}-11 \mathrm{PET}$ parameters with histopathology

The sensitivity and specificity of ${ }^{68} \mathrm{Ga}-\mathrm{PSMA}-11 \mathrm{PET}$ detecting pelvic lymph nodes metastasis were $40.6 \%$ and $96.8 \%$ (13/32 and 149/154, respectively). Of 94 pT $\geq 3$ a prostatic lesions, $45.7 \%(n=43)$ were detected (miT $\geq 3 a)$ by ${ }^{68} \mathrm{Ga}$-PSMA-11 PET. $80.4 \%(n=74)$ of pT2 prostatic lesions were correctly classified as miT2. Cross tables are presented in Supplementary Table 1 and Table 2.

In the univariate analysis (Supplementary Table 3) a significant association was detected between a positive surgical margin and the following parameters: high miT status (miT $\geq 3 a, O R: 3.38, p=0.004$ ), miN1 status (OR: 7.526, $p<0.001$ ), SUV $\max (\mathrm{OR}: 1.026, p=0.039$ ) and TL (OR: 1.007, $p=0.021)$. In the multivariate analysis (Table 4), miN1 (OR: $5.428, p=0.004$ ) was significantly associated with a positive surgical margin. Moreover, a significant association was present between $\mathrm{miT} \geq 3 \mathrm{a}$ and $\mathrm{GS} \geq 8$ (OR: 2.696, $p=0.003$ ) (Table 5).

Table 4. Multivariate analysis for the association of ${ }^{68}$ Ga-PSMA-11 PET findings with surgical margin status.

\begin{tabular}{lllll}
\multicolumn{1}{c}{ No. of patients } & Odds ratio & $95 \% \mathrm{Cl}$ & $p$ value* \\
\hline miTNM classification, no., $\mathrm{n}=186$ & & & \\
\hline miT status & 125 & Reference & & \\
\hline 2 & 61 & 2.065 & $0.802-5.315$ & 0.133 \\
\hline$\geq$ 3a & & & & \\
\hline miN status & 168 & Reference & & \\
\hline No LN metastasis & 18 & 5.428 & $1.708-17.249$ & 0.004 \\
\hline With LN metastasis & 183 & 1.015 & $0.988-1.044$ & 0.282 \\
\hline SUV & & & \\
\hline max & of prostatic lesions & & & \\
\hline TL of prostatic lesions & 183 & 1.004 & $0.998-1.011$ & 0.166
\end{tabular}

*Significant associations are given in bold.

$\mathrm{Cl}=$ confidence interval; $\mathrm{LN}=$ lymph node; $\mathrm{PET}$ = positron emission tomography; $\mathrm{PSMA}=$ prostatespecific membrane antigen; SUV = standardized uptake value; $T L=$ total lesion; $T V=$ tumor volume

Table 5. Univariate analysis for the association of ${ }^{68} \mathrm{Ga}$-PSMA-11 PET findings with Gleason Score. 
No. of Odds ratio $95 \% \mathrm{Cl} \quad p$ value*

patients

miTNM classification, no., $\mathrm{n}=186$

miT status

\begin{tabular}{|c|c|c|c|c|}
\hline 2 & 125 & Reference & & \\
\hline$\geq 3 a$ & 61 & 2.696 & $1.39-5.23$ & 0.003 \\
\hline \multicolumn{5}{|l|}{ miN status } \\
\hline No LN metastasis & 168 & Reference & & \\
\hline With LN metastasis & 18 & 2.187 & $0.812-5.887$ & 0.122 \\
\hline $\mathrm{SUV}_{\text {mean }}$ of prostatic lesions & 183 & 1.020 & $0.986-1.056$ & 0.248 \\
\hline $\mathrm{SUV}_{\max }$ of prostatic lesions & 183 & 1.017 & $0.995-1.040$ & 0.138 \\
\hline TV of prostatic lesions & 183 & 0.981 & $0.941-1.022$ & 0.353 \\
\hline TL of prostatic lesions & 183 & 1.006 & $1-1.012$ & 0.056 \\
\hline
\end{tabular}

*Significant associations are given in bold.

$\mathrm{Cl}=$ confidence interval; $\mathrm{LN}=$ lymph node; $\mathrm{PET}$ = positron emission tomography; $\mathrm{PSMA}$ = prostatespecific membrane antigen; SUV = standardized uptake value; $T L=$ total lesion; $T V=$ tumor volume.

\section{Predictors of BCR-free survival}

Kaplan-Meier curves for BCR-free survival with different clinicopathological and miTNM-derived parameters are shown in Fig.1-3 and Supplementary Fig.2.

The miTNM derived parameters miT2 vs. miT3 disease or higher (log-rank, $p<0.001$, Fig. 2A), miN0 vs. miN1/2 (log-rank, $p=0.005$, Fig. 2B) and miTNM stage group IA compared with miTNM stage group IB (log-rank, $p=0.022$, Fig. 1 ) were associated with significantly different BCR-free survival rate. Lower $\mathrm{SUV}_{\text {mean }}$ and $\mathrm{SUV}_{\text {max }}$ as quantitative parameters from ${ }^{68} \mathrm{Ga}$-PSMA-11 PET were also associated with longer BCR-free in patients (Fig. 3C and 3D, log-rank, $p=0.035, p=0.037$, respectively).

The following pathological features were associated with longer BCR-free survival: pT2 vs. $\geq \mathrm{pT} 3 \mathrm{a}$ (logrank, $p<0.001$, Supplementary Fig. 2A). pN0 vs. pN1 (log-rank, $p<0.001$, Supplementary Fig. 2B), lower Gleason Grades (GS<8) (log-rank, $p<0.001$, Supplementary Fig. $2 \mathrm{C}$ ) and negative surgical margins (logrank, $p<0.001$, Supplementary Fig. 2D).

Results from a univariate Cox regression analysis investigating preoperative and postoperative risk factors for BCR is presented in Table 6. We found that following factors were significantly associated 
with BCR-free survival in prostate cancer patients: clinical data including age (HR: $1.056,95 \%$ Cl: $1.018-$ 1.096, $p=0.004$ ) and initial PSA (iPSA) (HR: 1.021, 95\% Cl: 1.007-1.035, $p=0.003$ ); pathological data including Gleason score (GS $\geq 8$ vs. GS $<8, H R: 5.097,95 \%$ Cl: $3.013-8.625, p<0.001)$, pT stage (pT $\geq 3$ vs. pT<3, HR: 2.935, 95\% Cl: 1.665-5.173, $p<0.001$ ), pN stage (pN1 vs. pN0, HR: 3.378, 95\% Cl: 1.901-6, $p<0.001$ ) and surgical margin (positive vs. negative, HR: $3.421,95 \% \mathrm{Cl}: 1.890-6.193, p<0.001$ ). Imaging parameters including miT stage (miT $\geq 3$ a vs. miT $<3, \mathrm{HR}: 2.811,95 \% \mathrm{Cl}: 1.673-4.722, p<0.001$ ), miN stage (miN1 vs. miN0, HR: 2.691, 95\% Cl: 1.311-5.527, $p=0.007)$, SUV mean $_{\text {of }}$ prostatic lesions (HR: $1.019,95 \%$ Cl: 1.002-1.036, $p=0.028)$, SUV $\max$ of prostatic lesions (HR: $1.015,95 \% \mathrm{Cl}: 1.004-1.026, p=0.008)$ and TV of prostatic lesions (HR: 0.948, 95\% Cl: 0.909-0.988, $p=0.011$ ).

Table 6. Univariable analysis for the association of baseline factors with BCR-free survival. 
No. of Hazard ratio $95 \% \mathrm{Cl} \quad p$ value* patients

Clinical data

$\begin{array}{lcccc}\text { Age } & 186 & 1.056 & 1.018-1.096 & \mathbf{0 . 0 0 4} \\ \text { iPSA } & 184 & 1.021 & 1.007-1.035 & \mathbf{0 . 0 0 3}\end{array}$

Pathological data

Gleason score in surgical specimen, no., $n=186$

\begin{tabular}{|c|c|c|c|c|}
\hline $6-7$ & 133 & Reference & & \\
\hline $8-10$ & 53 & 5.097 & $3.013-8.625$ & $<0.001$ \\
\hline \multicolumn{5}{|l|}{ Pathological stage, no., $n=186$} \\
\hline \multicolumn{5}{|l|}{ pT status } \\
\hline 2 & 92 & Reference & & \\
\hline$\geq 3$ & 94 & 2.935 & $1.665-5.173$ & $<0.001$ \\
\hline \multicolumn{5}{|l|}{ pN status } \\
\hline 0 & 154 & Reference & & \\
\hline 1 & 32 & 3.378 & $1.901-6.000$ & $<0.001$ \\
\hline \multicolumn{5}{|l|}{ Surgical margin, no., $\mathrm{n}=180$} \\
\hline Negative & 152 & Reference & & \\
\hline Positive & 28 & 3.421 & $1.890-6.193$ & $<0.001$ \\
\hline \multicolumn{5}{|l|}{ Imaging parameters } \\
\hline \multicolumn{5}{|c|}{ miTNM classification, no., $\mathrm{n}=186$} \\
\hline \multicolumn{5}{|l|}{ miT status } \\
\hline 2 & 125 & Reference & & \\
\hline$\geq 3 a$ & 61 & 2.811 & $1.673-4.722$ & $<0.001$ \\
\hline \multicolumn{5}{|l|}{ miN status } \\
\hline No LN metastasis & 168 & Reference & & \\
\hline With LN metastasis & 18 & 2.691 & $1.311-5.527$ & 0.007 \\
\hline$S_{\text {SUV }}$ mean of prostatic lesions & 183 & 1.019 & $1.002-1.036$ & 0.028 \\
\hline
\end{tabular}




\begin{tabular}{|c|c|c|c|c|}
\hline$<$ median & 91 & Reference & & \\
\hline$\geq$ median & 92 & 1.752 & $1.030-2.981$ & 0.039 \\
\hline $\mathrm{SUV}_{\max }$ of prostatic lesions & 183 & 1.015 & $1.004-1.026$ & 0.008 \\
\hline \multicolumn{5}{|c|}{ SUV $_{\max }$ of prostatic lesions, no. $n=183$} \\
\hline$<$ median & 91 & Reference & & \\
\hline$\geq$ median & 92 & 1.744 & $1.025-2.968$ & 0.040 \\
\hline TV of prostatic lesions & 183 & 0.948 & $0.909-0.988$ & 0.011 \\
\hline \multicolumn{5}{|c|}{ TV of prostatic lesions, no., $\mathrm{n}=183$} \\
\hline$<$ median & 91 & Reference & & \\
\hline$\geq$ median & 92 & 0.987 & $0.587-1.661$ & 0.962 \\
\hline TL of prostatic lesions & 183 & 1.003 & $1.000-1.006$ & 0.072 \\
\hline \multicolumn{5}{|c|}{ TL of prostatic lesions, no., $\mathrm{n}=183$} \\
\hline$<$ median & 91 & Reference & & \\
\hline$\geq$ median & 92 & 0.957 & $0.568-1.612$ & 0.869 \\
\hline
\end{tabular}

*Significant associations are given in bold.

$\mathrm{BCR}=$ biochemical recurrence; $\mathrm{Cl}=$ confidence interval; iPSA = initial PSA; IQR = interquartile range; $\mathrm{LN}=$ lymph node; $\mathrm{PET}=$ positron emission tomography; $\mathrm{PSA}=$ prostate-specific antigen; $\mathrm{PSMA}=$ prostatespecific membrane antigen; SUV = standardized uptake value; $T L=$ total lesion; $T V=$ tumor volume.

In the multivariate Cox regression analysis (Table 7) the following factors were independent predictors for BCR-free survival: serum PSA level (HR: 1.024, 95\% Cl: 1.005-1.043, $p=0.014)$, advanced pathological Gleason Score (GS $\geq 8$ vs. GS<8, HR: 3.253, 95\% Cl: 1.779-5.950; $p<0.001)$ and miT stage (miT $\geq 3 a$ vs. miT<3, HR: 1.941, 95\% Cl: 1.047-3.599, $p=0.035)$.

Table 7. Multivariable analysis for the association of baseline factors with BCR-free survival. 
No. of Hazard ratio $95 \% \mathrm{Cl} \quad p$ value* $^{*}$ patients

Clinical data

Age, no., $\mathrm{n}=186$

Continuous

1.030

$0.991-1.071$

0.133

iPSA, no., $\mathrm{n}=184$

Continuous

1.024

$1.005-1.043 \quad 0.014$

Pathological data

Gleason score in surgical specimen, no., $\mathrm{n}=186$

\begin{tabular}{|c|c|c|c|c|}
\hline $6-7$ & 133 & Reference & & \\
\hline $8-10$ & 53 & 3.253 & $1.779-5.950$ & $<0.001$ \\
\hline \multicolumn{5}{|l|}{ pT status, no., $\mathrm{n}=186$} \\
\hline 2 & 92 & Reference & & \\
\hline 3 & 94 & 1.471 & $0.773-2.797$ & 0.239 \\
\hline \multicolumn{5}{|l|}{ pN status, no., $n=186$} \\
\hline No LN metastasis & 154 & Reference & & \\
\hline With LN metastasis & 32 & 1.027 & $0.418-2.525$ & 0.954 \\
\hline \multicolumn{5}{|c|}{ Surgical margin, no., $\mathrm{n}=180$} \\
\hline Negative & 152 & Reference & & \\
\hline Positive & 28 & 1.539 & 0.716-3.305 & 0.269 \\
\hline \multicolumn{5}{|l|}{ Imaging parameters } \\
\hline \multicolumn{5}{|c|}{ miT status from PSMA PET, no., $\mathrm{n}=186$} \\
\hline 2 & 125 & Reference & & \\
\hline$\geq 3 a$ & 61 & 1.941 & $1.047-3.599$ & 0.035 \\
\hline \multicolumn{5}{|c|}{ miN status from PSMA PET, no., $\mathrm{n}=186$} \\
\hline No LN metastasis & 168 & Reference & & \\
\hline With LN metastasis & 18 & 1.233 & $0.389-3.908$ & 0.722 \\
\hline \multicolumn{5}{|l|}{$S U V_{\text {mean }}$, no., $n=183$} \\
\hline Continuous & & 0.743 & $0.491-1.123$ & 0.159 \\
\hline
\end{tabular}


SUV max no., $\mathrm{n}=183$

Continuous

1.202

$0.943-1.532$

0.137

TV

Continuous

0.934

$0.883-0.988$

0.017

* Significant associations are given in bold.

$\mathrm{BCR}=$ biochemical recurrence; $\mathrm{Cl}=$ confidence interval; $\mathrm{PSSA}=$ initial PSA; $\mathrm{LN}=$ lymph node; $\mathrm{PET}=$ positron emission tomography; PSA = prostate-specific antigen; PSMA = prostate-specific membrane antigen; SUV = standardized uptake value; TV = tumor volume.

\section{Discussion}

Prognostic tools of BCR are required and essential for improving treatment management of prostate cancer patients and reducing prostate cancer-associated mortality of patients developing BCR after primary treatment [31]. With the successful application of PSMA-ligand PET for primary staging in prostate cancer patients, clinical studies are necessary to investigate its predictive value. PSMA-ligand PET is increasingly used for selection, monitoring and individualization of prostate cancer treatments.

The present analysis is the first to investigate the association of miTNM classification from preoperative ${ }^{68} \mathrm{Ga}$-PSMA-11 PET imaging and postoperative histopathological findings and the potential of miTNM reporting to serve as predictors for BCR after RP. Consequently, we performed a prognostic validation of the miTNM system as a framework for PSMA-ligand PET reporting in a relatively large patient cohort. Preoperative ${ }^{68} \mathrm{Ga}-\mathrm{PSMA}-11$ PET and miTNM classification could help to stratify risk for BCR after RP and could potentially further influence the clinical patient management.

Previous studies have extensively assessed the predictors of BCR. Clinicopathological characteristics, including pathological aggressive GS [5-9], positive nerve invasion [8], pathological T stage [7] and preoperative PSA [3-6] were proven to have a strong association with BCR. Our present results are in accordance with literature data. The data from our analysis outline serum PSA level (HR: 1.024, 95\% Cl: 1.005-1.043, $p=0.014$ ) and advanced pathological Gleason score (GS $\geq 8$ vs. $G S<8, \mathrm{HR}: 3.253,95 \% \mathrm{Cl}$ : $1.779-5.950 ; p<0.001)$ as important histopathological predictors of BCR.

In addition, and novel compared to the current literature our data indicate that also parameters from ${ }^{68} \mathrm{Ga}-$ PSMA-11 PET might serve as non-invasive biomarkers. We identified a miT stage $\geq 3 a$ in ${ }^{68} \mathrm{Ga}-\mathrm{PSMA}-11$ PET as surrogate for higher GS (OR: $2.696,95 \% \mathrm{Cl}: 1.39-5.23, p=0.003)$ and worse BCR-free survival (HR: $1.941,95 \% \mathrm{Cl}: 1.047-3.599, p=0.035)$. Notably, pelvic lymph node metastases in ${ }^{68} \mathrm{Ga}-\mathrm{PSMA}-11 \mathrm{PET}$ were not detected as an independent predictor for BCR in this study (HR: 1.233, 95\% Cl: 0.389-3.908, $p=0.722$ ). However, the BCR-free survival differed significantly between miN0 and miN1 group (log-rank, $p=0.005$ ). Interestingly also Raheem et al. have failed to detect lymph nodes in histopathology as an independent 
predictor to BCR after RP in a study including 359 patients [4]. Of note, the sample size of miN1 group $(n=18)$ in our cohort was relatively small. Thus, the interpretation of results should be with caution, and further studies including more miN1 patients are needed to clarify the predictive value of miN classification for BCR after RP.

Besides, our results indicate a negative association of TV with BCR-free survival (HR: $0.934,95 \% \mathrm{Cl}$ : $0.883-0.988, p=0.017)$. Contrarily, Choi et al. have reported a significantly higher BCR-free survival rate in PT2 prostate cancer patients with percent tumor volume $\leq 7.5 \%$, which was assessed using histological samples $(p<0.001)$ [32]. This is partly related to the methods of obtaining tumor volume and studies are necessary to assess the standard of TV calculation from ${ }^{68} \mathrm{Ga}$-PSMA-11 PET and pathological samples.

With this work we also introduced a stage grading system based on the recent proposed molecular staging system (miTNM staging system, version 1.0) combined with quantitative parameters. It is intended to mirror the AJCC staging system based on clinicopathological parameters which has proven to be a fundamental tool that also informs treatment decisions [33]. Bhindi et al. has confirmed the ability of the $8^{\text {th }}$ edition to predict oncologic outcomes [34]. However, the AJCC staging system utilized clinical or pathological TNM stage and no parameters from imaging. With the increasing use of PSMA-ligand PET in clinical routine, a logical next step is to use information from non-invasive imaging prior to definite treatment for risk stratification.

We have shown that the miT stage is an independent predictor of $B C R$, and we observed a widely varying prognosis in the miT2 stage patients. Similarly, a recent study has revealed that high intraprostatic ${ }^{68} \mathrm{Ga}$ PSMA-11 uptake (SUV $\max >8$ ) predicts short progression-free survival rate among patients with $G S 3+4$ on biopsy [35]. The significant difference in BCR-free survival rate has been confirmed in IA and IB stage groups. Our findings propose that a SUV $V_{\max }$ cut-off extracted from literature could further stratify the group of miT2 primary disease into patients with more aggressive disease and worse prognosis. Further studies are necessary for prognostic validation of other stage groups.

The present study has several limitations. It is a retrospective analysis and includes only patients from a single center, which can introduce potential bias. Despite inclusions of a large number of patients the sample size of patients in the miM1 group was too small to conduct meaningful analysis. This is mainly related to the fact that most patients with extrapelvic metastases do not undergo primary curative RP but either get systemic treatment with or without local treatment. This explains the low number of patients in miTNM stage group III and IV. In summary, further prospective investigations with large patient numbers are necessary to fully investigate the potential of the miTNM staging and our proposed grading system to predict patient outcome after curative intent RP.

\section{Conclusion}

Our retrospective analysis indicates that the miTNM framework developed to standardize PSMA-ligand PET reported is independently associated with BCR-free survival of primary prostate cancer after RP. We 
demonstrated significant associations between ${ }^{68} \mathrm{Ga}$-PSMA-11 PET findings and histopathological parameters. In summary, our results outline that the miTNM classification and the presented further development of a miTNM based stage group system can serve as non-invasive imaging biomarkers of risk stratification for primary prostate cancer patients. However, further and prospective studies including patients with different treatments and stages are needed to fully assess the predictive value of PSMAligand PET imaging in the setting of newly diagnosed prostate cancer.

\section{Abbreviations}

${ }^{68}$ Ga: Gallium-68; AJCC: American Joint Committee on Cancer; BCR: biochemical recurrence; BSI: bone scan index; Cl: confidence interval; CT: computed tomography; DNA: deoxyribonucleic acid; ESCC: esophageal squamous cell carcinoma; FDG: fluorodeoxyglucose; GS: Gleason score; HR: hazard ratio; iPSA: initial PSA; IQR: interquartile range; mCRPC: metastatic castration-resistant prostate cancer; miTNM: imaging TNM classification; MRI: magnetic resonance imaging; OR: odds ratio; PET: positron emission tomography; PSA: prostate-specific antigen; PSMA: prostate-specific membrane antigen; RP: radical prostatectomy; SD: standard deviation; SUV: standardized uptake value; TL: total lesion; TV: tumor volume; VOI: volume of interest.

\section{Declarations}

\section{Ethics approval and informed consent}

All procedures performed in studies involving human participants were in accordance with the Declaration of Helsinki. This retrospective study has been approved by the Ethics Committee of the Technical University Munich (750/20 S-KH).

\section{Consent for publication}

Not applicable.

\section{Availability of data and materials}

The datasets generated during and/or analyzed during the current study are available from the corresponding author on a reasonable request

\section{Competing interests}

The authors declare that they have no competing interests.

\section{Funding}

Matthias Eiber, Hans-Juergen Wester and Katja Steiger received funding from the SFB 824 (DFG Sonderforschungsbereich 824, Project B11 and Z2) from the Deutsche Forschungsgemeinschaft, Bonn, 
Germany. Hui Wang received a scholarship from the China Scholarship Council (CSC).

\section{Acknowledgments}

Not applicable.

\section{Authors' contributions}

HW was involved in study design, data acquisition, methodology and manuscript writing. TA was involved in data acquisition. CW supported statistical analysis. TL, KS, IR, TH, TM, KK, HJW, WW supported the data collection and revised the manuscript. ME was involved in study design, data acquisition and critical manuscript revising. All authors read and approved the final manuscript.

\section{Author's information}

${ }^{1}$ Department of Nuclear Medicine, Klinikum rechts der Isar, Technical University Munich, Ismaninger Str. 22, 81675 Munich, Germany. ${ }^{2}$ Department of Urology, Klinikum rechts der Isar, Technical University Munich, Ismaninger Str. 22, 81675 Munich, Germany. ${ }^{3}$ Martini-Klinik Prostate Cancer Center, University Hospital Hamburg-Eppendorf, Martinistr. 52, 20246 Hamburg, Germany. ${ }^{4}$ Institute of Pathology, School of Medicine, Technical University Munich, Trogerstr. 18, 81675, Munich, Germany. ${ }^{5}$ Department of Urology, University Hospital Hamburg-Eppendorf, Martinistr. 52, 20246 Hamburg, Germany. 6 Pharmaceutical Radiochemistry, Technical University of Munich, Walther-Meißner-Str. 3, 85748 Garching, Germany

\section{References}

1. Schwarzenboeck SM, Rauscher I, Bluemel C, Fendler WP, Rowe SP, Pomper MG, et al. PSMA Ligands for PET Imaging of Prostate Cancer. J Nucl Med. 2017;58(10):1545-1552.

2. Paller CJ, Antonarakis ES, Eisenberger MA, Carducci MA. Management of patients with biochemical recurrence after local therapy for prostate cancer. Hematol Oncol Clin North Am. 2013;27(6):1205-1219, viii.

3. Qi P, Tsivian M, Abern MR, Bañez LL, Tang P, Moul JW, et al. Long-term oncological outcomes of men undergoing radical prostatectomy with preoperative prostate-specific antigen $<2.5 \mathrm{ng} / \mathrm{ml}$ and $2.5-4$ ng/ml. Urol Oncol. 2013;31(8):1527-1532.

4. Abdel Raheem A, Chang KD, Alenzi MJ, Ham WS, Han WK, Choi YD, et al. Predictors of biochemical recurrence after Retzius-sparing robot-assisted radical prostatectomy: Analysis of 359 cases with a median follow-up period of 26 months. Int J Urol. 2018;25(12):1006-1014.

5. Freedland SJ, Presti JC, Amling CL, Kane CJ, Aronson WJ, Dorey F, et al. Time trends in biochemical recurrence after radical prostatectomy: results of the SEARCH database. Urology. 
6. Aoun F, Albisinni S, Henriet B, Tombal B, Van Velthoven R, Roumeguère T. Predictive factors associated with biochemical recurrence following radical prostatectomy for pathological T2 prostate cancer with negative surgical margins. Scand J Urol. 2017;51(1):20-26.

7. Inagaki T, Kohjimoto Y, Nishizawa S, Kuramoto T, Nanpo Y, Fujii R, et al. PSA at postoperative three months can predict biochemical recurrence in patients with pathological T3 prostate cancer following radical prostatectomy. Int J Urol. 2009;16(12):941-946.

8. Xu X, Li Q, Chang C, Wang X, Xie L. Metabolic Syndrome Is Not Associated With Prostate Cancer Recurrence: A Retrospective Analysis of a Chinese Cohort. Front Oncol. 2020;10:63.

9. Cao D, Kibel AS, Gao F, Tao Y, Humphrey PA. The Gleason Score of Tumor at the Margin in Radical Prostatectomy is Predictive of Biochemical Recurrence. Am J Surg Pathol. 2010;34(7):994-1001.

10. Maurer T, Gschwend JE, Rauscher I, Souvatzoglou M, Haller B, Weirich G, et al. Diagnostic Efficacy of ${ }^{68}$ Gallium-PSMA Positron Emission Tomography Compared to Conventional Imaging for Lymph Node Staging of 130 Consecutive Patients with Intermediate to High Risk Prostate Cancer. J Urol. 2016;195(5):1436-1443.

11. Fendler WP, Calais J, Eiber M, Flavell RR, Mishoe A, Feng FY, et al. Assessment of 68Ga-PSMA-11 PET Accuracy in Localizing Recurrent Prostate Cancer: A Prospective Single-Arm Clinical Trial. JAMA Oncol. 2019;5(6):856-863.

12. Hofman MS, Lawrentschuk N, Francis RJ, Tang C, Vela I, Thomas P, et al. Prostate-specific membrane antigen PET-CT in patients with high-risk prostate cancer before curative-intent surgery or radiotherapy (proPSMA): a prospective, randomised, multicentre study. Lancet. 2020;395(10231):12081216.

13. Silver DA, Pellicer I, Fair WR, Heston WD, Cordon-Cardo C. Prostate-specific membrane antigen expression in normal and malignant human tissues. Clin Cancer Res. 1997;3(1):81-85.

14. Cimadamore A, Cheng M, Santoni M, Lopez-Beltran A, Battelli N, Massari F, et al. New Prostate Cancer Targets for Diagnosis, Imaging, and Therapy: Focus on Prostate-Specific Membrane Antigen. Front Oncol. 2018;8:653.

15. Mannweiler S, Amersdorfer P, Trajanoski S, Terrett JA, King D, G M. Heterogeneity of prostatespecific membrane antigen (PSMA) expression in prostate carcinoma with distant metastasis. Pathol Oncol Res. 2009;15(2):167-172.

16. Minner S, Wittmer C, Graefen M, Salomon G, Steuber T, Haese A, et al. High level PSMA expression is associated with early PSA recurrence in surgically treated prostate cancer. Prostate. 
17. Ross JS, Sheehan CE, Fisher HA, Kaufman RP, Jr., Kaur P, Gray K, et al. Correlation of primary tumor prostate-specific membrane antigen expression with disease recurrence in prostate cancer. Clin Cancer Res. 2003;9(17):6357-6362.

18. Paschalis A, Sheehan B, Riisnaes R, Rodrigues DN, Gurel B, Bertan C, et al. Prostate-specific Membrane Antigen Heterogeneity and DNA Repair Defects in Prostate Cancer. Eur Urol. 2019;76(4):469478.

19. Wieder HA, Brücher BL, Zimmermann F, Becker K, Lordick F, Beer A, et al. Time course of tumor metabolic activity during chemoradiotherapy of esophageal squamous cell carcinoma and response to treatment. J Clin Oncol. 2004;22(5):900-908.

20. Armstrong AJ, Kaboteh R, Carducci MA, Damber JE, Stadler WM, Hansen M, et al. Assessment of the bone scan index in a randomized placebo-controlled trial of tasquinimod in men with metastatic castration-resistant prostate cancer (mCRPC). Urol Oncol. 2014;32(8):1308-1316.

21. Gafita A, Bieth M, Krönke M, Tetteh G, Navarro F, Wang H, et al. qPSMA: semiautomatic software for whole-body tumor burden assessment in prostate cancer using 68Ga-PSMA11 PET/CT. J Nucl Med. 2019;60(9):1277-1283.

22. Gafita A, Calais J, Hui W, Weber M, Rathke H, Esfandiari R, et al. Predictive factors and prediction nomograms for LuPSMA radioligand therapy in patients with metastatic castration-resistant prostate cancer: an international multicentre retrospective study. J Nucl Med. 2020;61(supplement 1):593-593.

23. Eiber M, Herrmann K, Calais J, Hadaschik B, Giesel FL, Hartenbach M, et al. Prostate Cancer Molecular Imaging Standardized Evaluation (PROMISE): Proposed miTNM Classification for the Interpretation of PSMA-Ligand PET/CT. J Nucl Med. 2018;59(3):469-478.

24. Eder M, Schäfer M, Bauder-Wüst U, Hull WE, Wängler C, Mier W, et al. ${ }^{68}$ Ga-complex lipophilicity and the targeting property of a urea-based PSMA inhibitor for PET imaging. Bioconjug Chem. 2012;23(4):688-697.

25. Martin R, Jüttler S, Müller M, Wester H-J. Cationic eluate pretreatment for automated synthesis of $\left[{ }^{68} \mathrm{Ga}\right] \mathrm{CPCR}$. 2. Nuclear medicine and biology. 2014;41(1):84-89.

26. Souvatzoglou M, Eiber M, Martinez-Moeller A, Fürst S, Holzapfel K, Maurer T, et al. PET/MR in prostate cancer: technical aspects and potential diagnostic value. Eur J Nucl Med Mol Imaging. 2013;40 Suppl 1:S79-88.

27. Eiber M, Maurer T, Souvatzoglou M, Beer AJ, Ruffani A, Haller B, et al. Evaluation of Hybrid $\triangle \otimes G a-$ PSMA Ligand PET/CT in 248 Patients with Biochemical Recurrence After Radical Prostatectomy. J Nucl 
Med. 2015;56(5):668-674.

28. Hofman MS, Hicks RJ, Maurer T, Eiber M. Prostate-specific Membrane Antigen PET: Clinical Utility in Prostate Cancer, Normal Patterns, Pearls, and Pitfalls. Radiographics. 2018;38(1):200-217.

29. Edge SB, Byrd DR, Carducci MA, Compton CC, Fritz A, Greene F. AJCC cancer staging manual: Springer New York; 2010.

30. Hoffmann MA, Miederer M, Wieler HJ, Ruf C, Jakobs FM, Schreckenberger M. Diagnostic performance of ${ }^{68}$ Gallium-PSMA-11 PET/CT to detect significant prostate cancer and comparison with ${ }^{18}$ FEC PET/CT. Oncotarget. 2017;8(67):111073-111083.

31. Brockman JA, Alanee S, Vickers AJ, Scardino PT, Wood DP, Kibel AS, et al. Nomogram Predicting Prostate Cancer-specific Mortality for Men with Biochemical Recurrence After Radical Prostatectomy. Eur Urol. 2015;67(6):1160-1167.

32. Choi SY, Chi BH, Lim B, Kyung YS, You D, Jeong IG, et al. Percent tumor volume vs American Joint Committee on Cancer staging system subclassification for predicting biochemical recurrence in patients with pathologic T2 prostate cancer. J Cancer Res Clin Oncol. 2020;146(2):537-543.

33. Buyyounouski MK, Choyke PL, McKenney JK, Sartor O, Sandler HM, Amin MB, et al. Prostate cancer - major changes in the American Joint Committee on Cancer eighth edition cancer staging manual. CA Cancer J Clin. 2017;67(3):245-253.

34. Bhindi B, Karnes RJ, Rangel LJ, Mason RJ, Gettman MT, Frank I, et al. Independent validation of the American Joint Committee on Cancer 8th edition prostate cancer staging classification. J Urol. 2017;198(6):1286-1294.

35. Roberts MJ, Morton A, Donato P, Kyle S, Pattison DA, Thomas P, et al. ${ }^{68}$ Ga-PSMA PET/CT tumour intensity pre-operatively predicts adverse pathological outcomes and progression-free survival in localised prostate cancer. Eur J Nucl Med Mol Imaging. 2021;48(2):477-482.

\section{Figures}




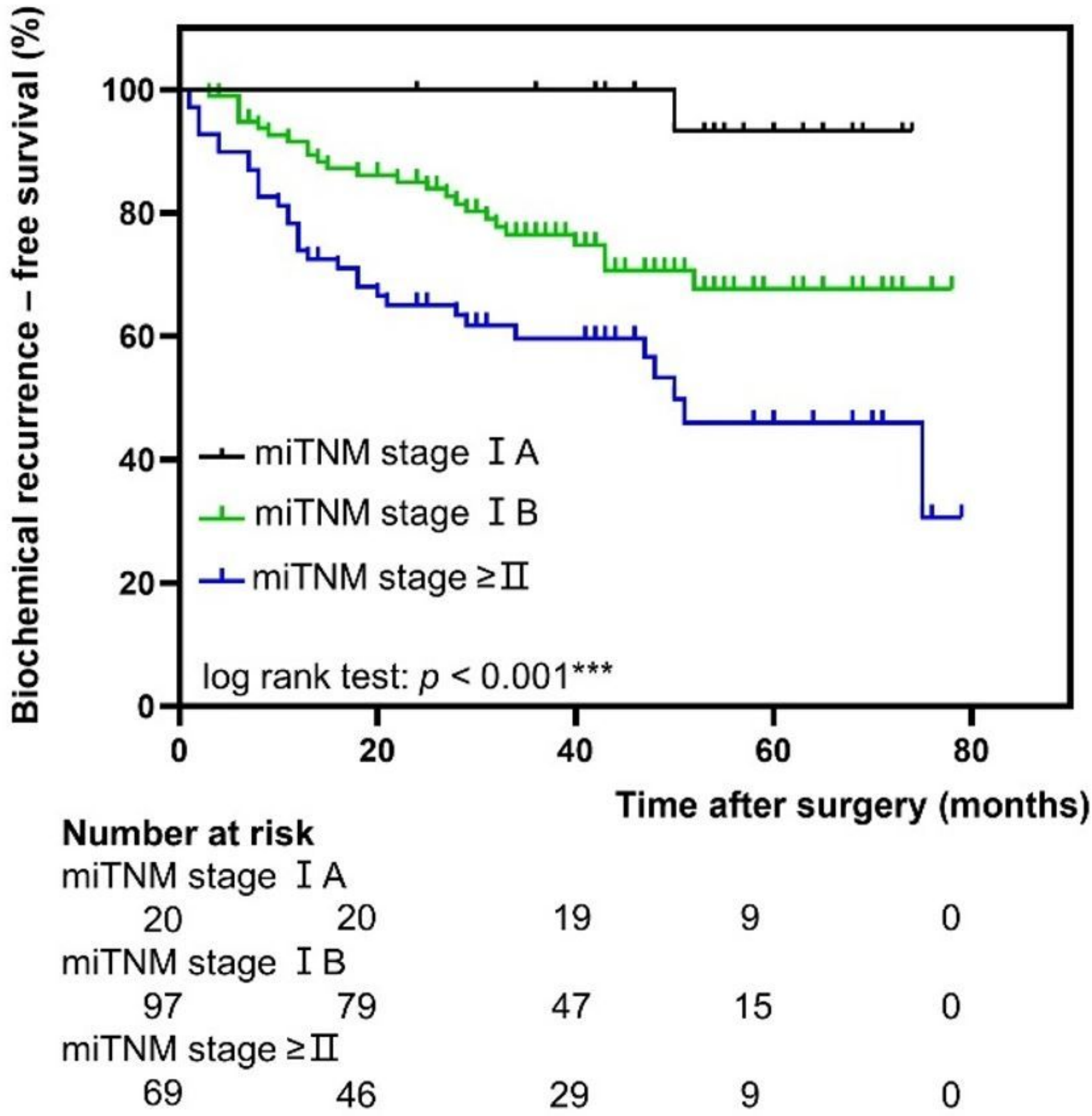

Figure 1

Biochemical recurrence-free survival according to miTNM stage. Pairwise comparison: miTNM stage IA vs. miTNM stage IB, $p=0.022$; miTNM stage IA vs. miTNM stage $\geq I I, p=0.001 ;$ miTNM stage IB vs. miTNM stage $\geq I l, p=0.005$. 

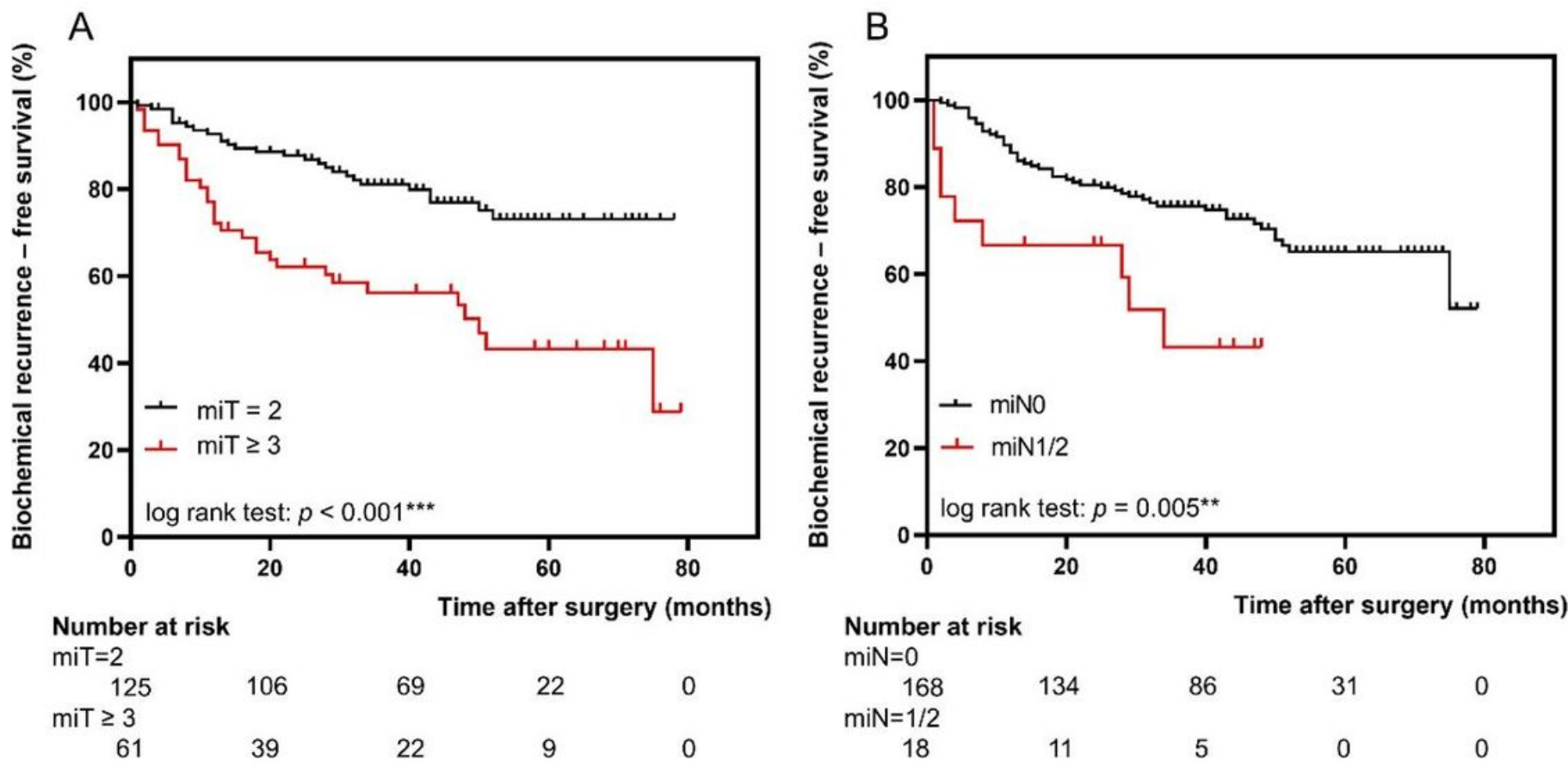

Figure 2

Longer biochemical recurrence-free survival was associated with $(A) \mathrm{miT}=2$ and $(B) \mathrm{miN}=0$. 

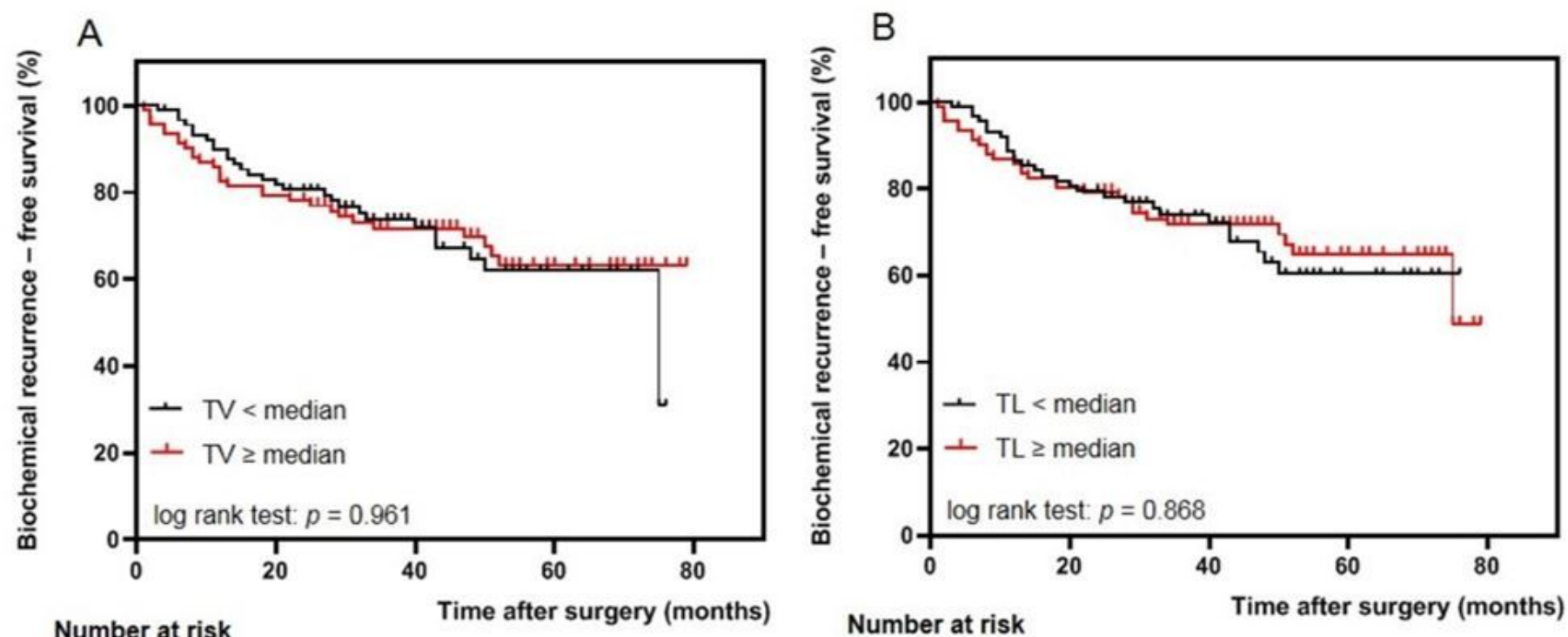

$\begin{array}{ccccc}\text { TV }<\text { median } \\ 91 & 72 & 39 & 13 & 0 \\ \text { TV } \geq \text { median } & & & & \\ 92 & 73 & 51 & 19 & 0\end{array}$
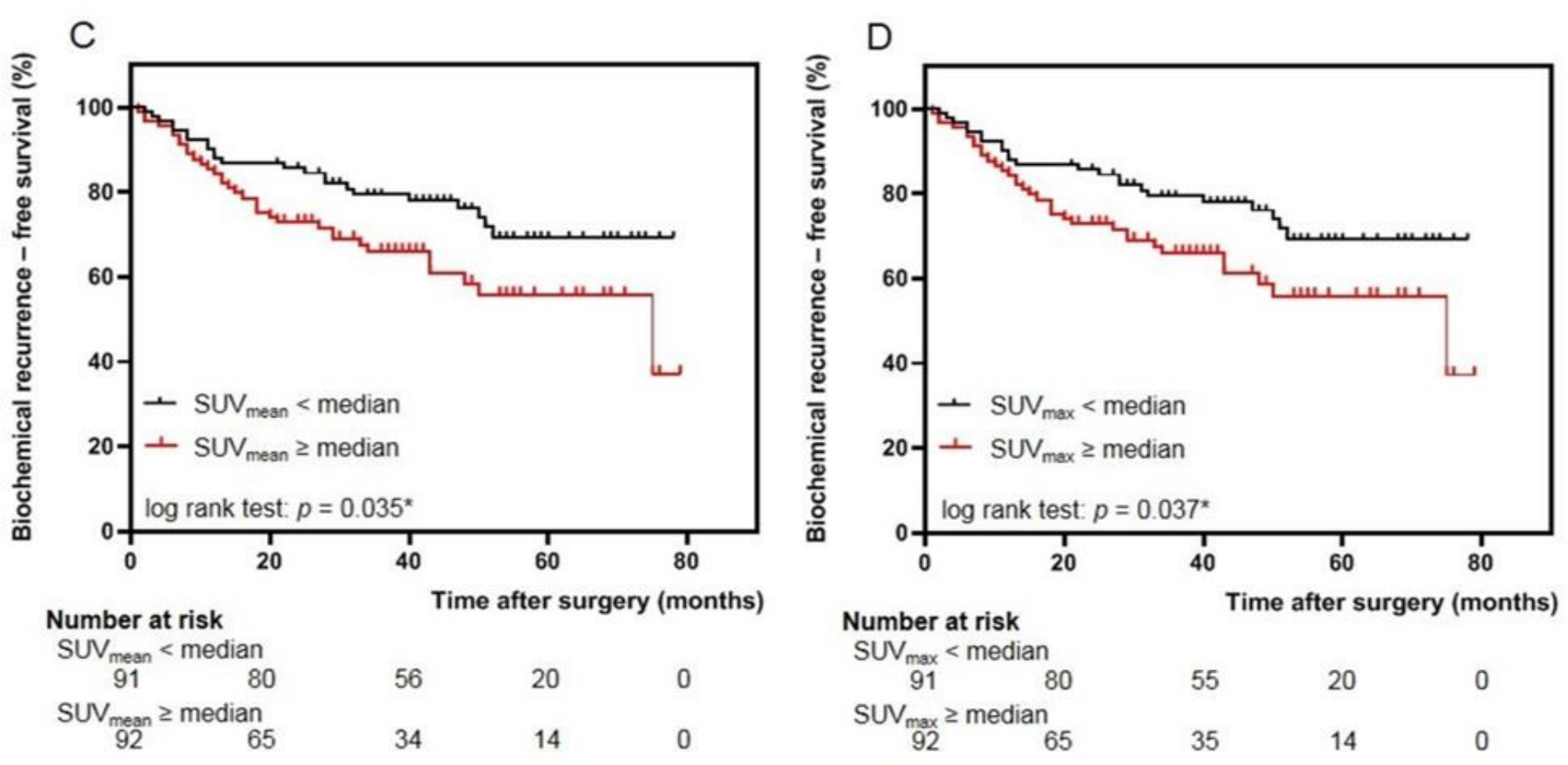

Figure 3

Kaplan-Meier curves comparing biochemical recurrence-free survival of selected patients stratified by (A) tumor volume, (B) total lesion, (C) SUVmean and (D) SUVmax. Longer biochemical recurrence-free survival was associated with lower SUVmean and SUVmax.

\section{Supplementary Files}

This is a list of supplementary files associated with this preprint. Click to download. 
- supplementarymaterialsBCR3notrackchange.docx 